\section{Tobacco use and second-hand tobacco smoke increase myocardial infarction risk}

INTERHEART, a standardized case-control study of acute myocardial infarction (AMI) including 27,089 participants from 52 countries, has assessed the risks associated with tobacco use and exposure to second-hand tobacco smoke.

Teo et al. found that current smokers were at a greater risk of nonfatal AMI (odds ratio [OR] 2.95, 95\% Cl 2.77-3.14; $P<0.0001$ ) than individuals who had never smoked. This risk increased by $5.6 \%$ for every additional cigarette smoked per day. Within 3 years of giving up cigarettes, the OR associated with former smoking fell to 1.87 (95\% Cl 1.55-2.24), although there was a residual excess risk for $>20$ years after quitting (OR 1.22, 95\% Cl 1.09-1.37). Use of chewing tobacco alone was associated with an OR for nonfatal AMI of 2.23 (95\% Cl 1.41-3.52), and smokers who also chewed tobacco had the highest increase in risk (OR 4.09, 95\% Cl 2.98-5.61). Individuals who had never smoked but who were exposed to second-hand smoke had an increased nonfatal AMI risk that ranged from $1.24(95 \% \mathrm{Cl}$ 1.17-1.32) in those exposed for $1-7 \mathrm{~h} /$ week to $1.62(95 \% \mathrm{Cl} 1.45-1.81)$ in those exposed for $>21 \mathrm{~h} /$ week. Overall, the highest population attributable risk was seen in young male current smokers (58.3\%, 95\% Cl 55.0-61.6). For individuals who had never smoked, the population attributable risk for exposure to secondhand smoke for $>1 \mathrm{~h} /$ week was $15.4 \%$ (95\% Cl 12.1-19.3).

Tobacco use is, therefore, one of the most important causes of AMI globally, particularly in men. The authors recommend that all forms of tobacco use should be discouraged and inhalation of second-hand smoke avoided.

Original article Teo KK et al. on behalf of the INTERHEART Study Investigators (2006) Tobacco use and risk of myocardial infarction in 52 countries in the INTERHEART study: a casecontrol study. Lancet 368: 647-658

\section{Ongoing risk factors for post-heart-transplant patients}

To date, the majority of investigations into the risk factors associated with cardiac transplantation have focused on the relationship between pretransplant risks and outcomes. A large-scale, multicenter, retrospective review by Kobashigawa and co-workers assessed ongoing post-transplant risk factors affecting the 5-year outcomes of heart transplant recipients. The authors conclude that ongoing risk factors affect post-transplant outcomes, and suggest that modification of these factors would minimize the occurrence of post-transplant complications.

The study reviewed 280 heart transplant patients who had survived $>1$ year, from 6 US transplant centers. Post-transplant renal dysfunction, measured as elevated serum creatinine level, was shown to significantly increase the risk of graft death (relative risk [RR] 1.59; $P<0.001)$. High total cholesterol levels and diabetes were associated with an increase in nonfatal major adverse cardiac events (RR 4.34, $P=0.01$ and RR 3.96, $P=0.02$, respectively). $\mathrm{BMI} \geq 33 \mathrm{~kg} / \mathrm{m}^{2}$ was identified as a risk factor for graft death, but was not significant (RR 1.80; $P=0.07$ ). None of the covariates examined was a significant predictor of cardiac allograft vasculopathy.

The authors note that the reviewed patients represent a low-risk group, and recognize that their study could have been underpowered to detect associations between certain risk factors and outcomes. The results indicate, however, that randomized trials investigating the effect of risk factor modification, for example tight control of diabetes, could improve the post-transplant management of heart recipients.

Original article Kobashigawa JA et al. (2006) Multicenter retrospective analysis of cardiovascular risk factors affecting long-term outcome of de novo cardiac transplant recipients. J Heart Lung Transplant 25: 1063-1069

\section{Cardiovascular function significantly improved by surgical repair of pectus excavatum}

Pectus excavatum (PE) is relatively common and accounts for approximately $90 \%$ of congenital chest wall deformities. Despite known underlying problems associated with $\mathrm{PE}$, however, including dyspnea, fatigue and tachycardia, there is little consensus as to whether surgical repair of the chest wall is anything other than a cosmetic procedure in these patients.

To assess whether surgical repair improves cardiac function, Malek and colleagues performed a meta-analysis of studies referenced between January 1960 and May 2005, identified 